\title{
Skin Manifestations of Endocrine Diseases
}

\author{
Cuyan DEMIRKESEN
}

Department of Pathology, i̇stanbul University, Cerrahpaşa Medical Faculty, ISTANBUL, TURKEY

\section{ABSTRACT}

Endocrine diseases may result in changes in cutaneous function and morphology, which cause various skin manifestations, including nonspecific or pathognomonic signs. Some of these manifestations are already known dermatologic diseases with only increased frequency in this patient group. As a result the skin may the play role of a screen displaying endocrine disorders, either due to hormone excess or deficiency. Awareness of the skin manifestations may permit prompt and adequate approach to the patients, and therefore facilitate the early diagnosis of the endocrine disease and even be life saving. Some of these manifestations may be recognized clinically, but sometimes they need to be confirmed histopathologically. In this article, many endocrine diseases and their associated skin lesions will be reviewed briefly.

Key Words: Skin, Pathology, Endocrine diseases

Endocrine diseases may result in skin manifestations, either due to hormone excess or deficiency (Table I). Some of them are already known dermatologic diseases with only increased frequency in this patient group (1). They can be recognized clinically, but sometimes they need to be confirmed histopathologically. The microscopic findings of the most frequent skin manifestations will be summarized in this article.

\section{ACANTHOSIS NIGRICANS}

Acanthosis nigrigans may be present in patients with acromegaly, hyperprolactinemia, diabetes mellitus (DM), Cushing's syndrome, and excessive androgen production. The skin, mostly in the axillae and nape of the neck becomes dark, soft, and velvet-like with delicate folds. The biopsy reveals hyperkeratosis and papillomatosis with slight acanthosis. The valleys between the papillae are filled with keratotic material (Figure 1). Slight hyperpigmentation of the basal cell layer can be seen, but the dark color of the lesion is due to hyperkeratosis rather than melanin.

\section{ACNE}

As a manifestations of Cushing's syndrome, acne can be seen together with skin thinning, purpura, hirsutism and striae distensea. The acneiform lesions associated with hypercorticism, are commonly seen on the face, neck, and shoulders. Comedones or cysts are rare, thus differing from acne vulgaris. On the other hand, a common clinical finding of hyperandrogenemia in women is severe acne vulgaris, accompanying thickened and coarse skin, the enlargement of pores on the face, excessive oiliness. Acne

(Turk Patoloji Derg 2015, 31(Suppl):145-154)

Received : 28.05.2015 Accepted : 01.06.2015 vulgaris may also develop in hyperprolactinemia. In the microscopic examination of acneiform lesions, the formation of comedone due to dilatation and thinning of the follicular epithelium and a keratotic plug with some sebaceous material are the characteristic findings.

\section{ACQUIRED PERFORATING DERMATOSIS}

Acquired perforating dermatosis has been reported in 10\% of diabetic patients (2). Most of these diabetic patients have chronic renal failure due to diabetic nephropathy (3). It is seen as umbilicated, hyperkeratotic papules or nodules on the lower and upper extremities, sometimes in a linear fashion. The Koebner phenomenon may be observed. Histopathologically, there is an epidermal invagination filled with keratotic plug, admixed with cellular debris, degenerated collagen or elastic fibers and inflammatory cells due to transepidermal elimination. There are vertically oriented coarse collagen bundles at the base of the invagination (Figure 2). Masson trichrome staining shows the typical transepidermal elimination of the collagen fibers. Elastica van Gieson or Orcein stains are used to demonstrate the collagen and elastic fibers within the debris. Some of these lesions may be associated with hair follicles and are called perforating folliculitis. Thickening of the vessel walls, evident with Periodic acid-Shiff (PAS) staining, may be seen in the upper dermis (4).

\section{ALOPECIA}

Various types of alopecia may be seen in some endocrine diseases. Elevated prolactin levels may cause hyperandrogenism due to the enhanced end-organ response

Correspondence: Cuyan DEMIRKESEN

İstanbul Üniversitesi, CerrahpaşaTıp Fakültesi, Patoloji Anabilim Dalı

Cerrahpaşa 34098, Fatih, İSTANBUL, TURKEY

E-mail: cdemirkesen@yahoo.com Phone: +90 5332569267 
Table I: Skin lesions showing association with endocrinopathies

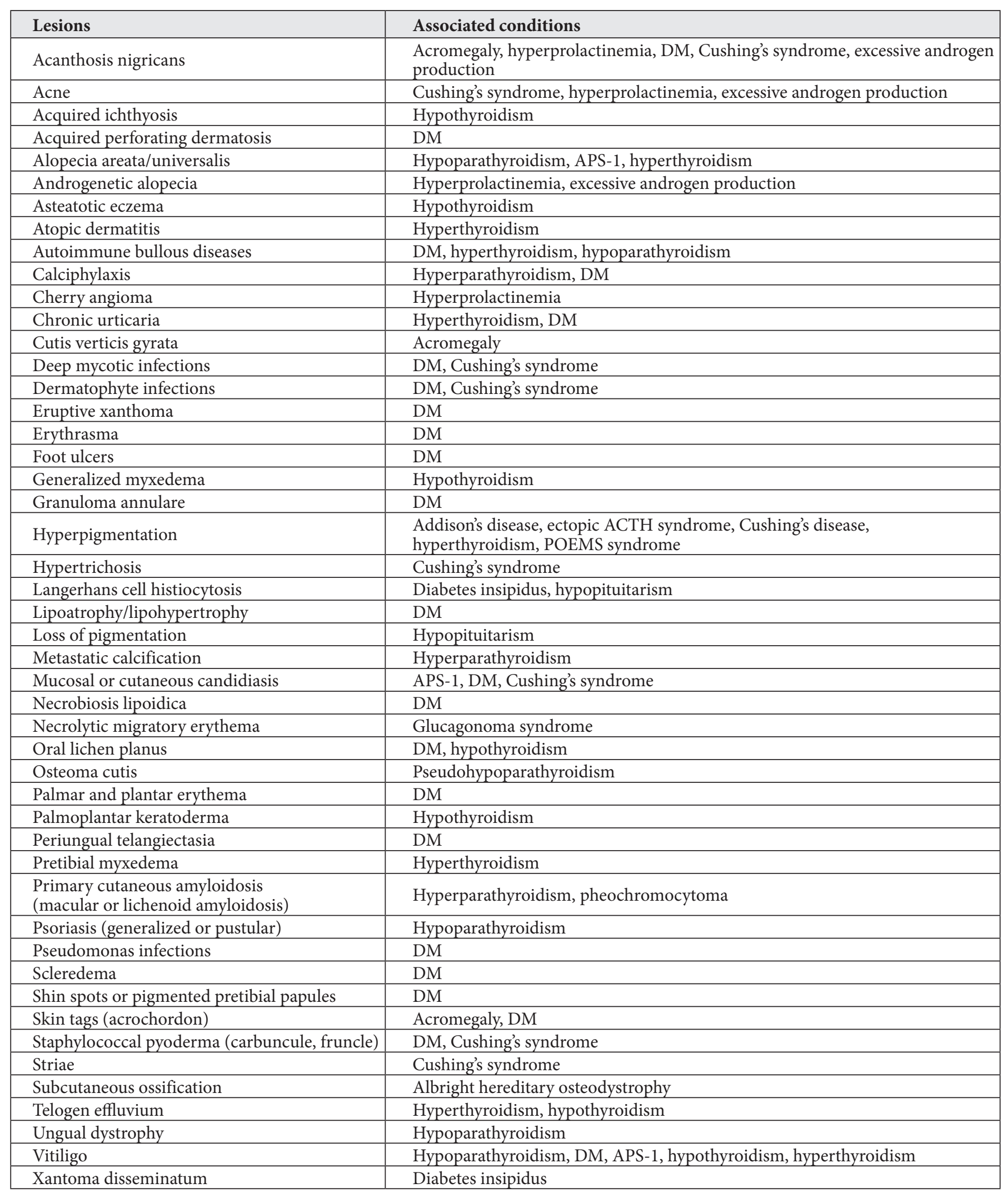

APS: Autoimmune polyglandular syndrome, DM: Diabetes mellitus. 
to normal androgen levels, resulting in androgenetic alopecia. Histopathologically, androgenetic alopecia is characterized by progressive diminution of follicular size (5). This results in a mixture of hairs with various bulb depth and shaft diameter. The normal ratio of terminal to vellus hairs should be at least $2: 1$, but in androgenetic alopecia, there is a reduction in this ratio, resulting in an increased number of vellus and indeterminate hairs which eventually surpass the number of terminal hairs (Figure 3). In hyperthyroidism, there may be diffuse scalp hair loss in some patients, as a result of alopecia areata or telogen effluvium. Alopecia areata is clinically characterized by sudden onset of patches of hair loss. It may undergo

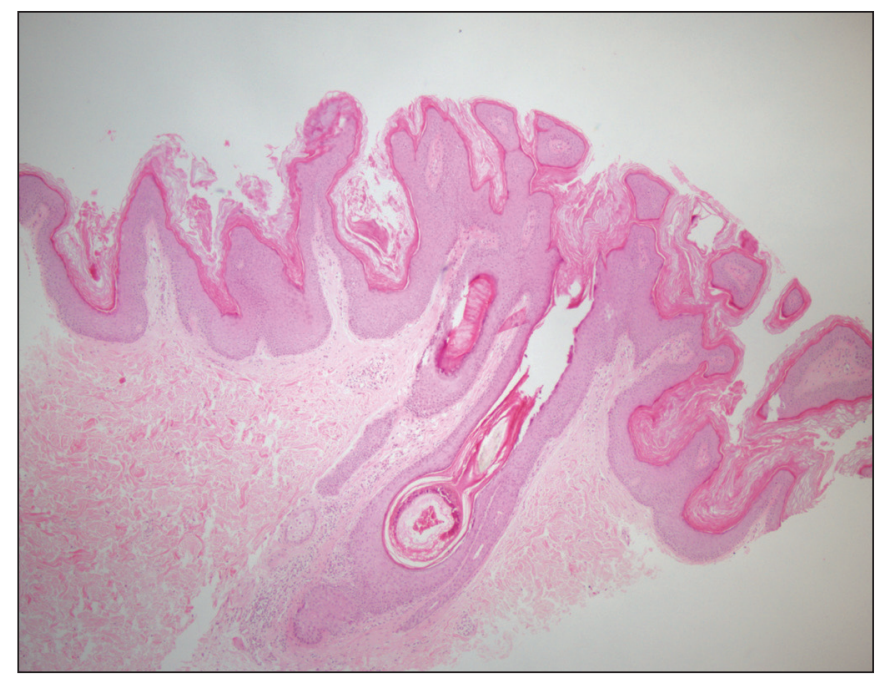

Figure 1: Acanthosis nigricans: Prominent hyperkeratosis and papillomatosis with mild acanthosis (HE; x40).

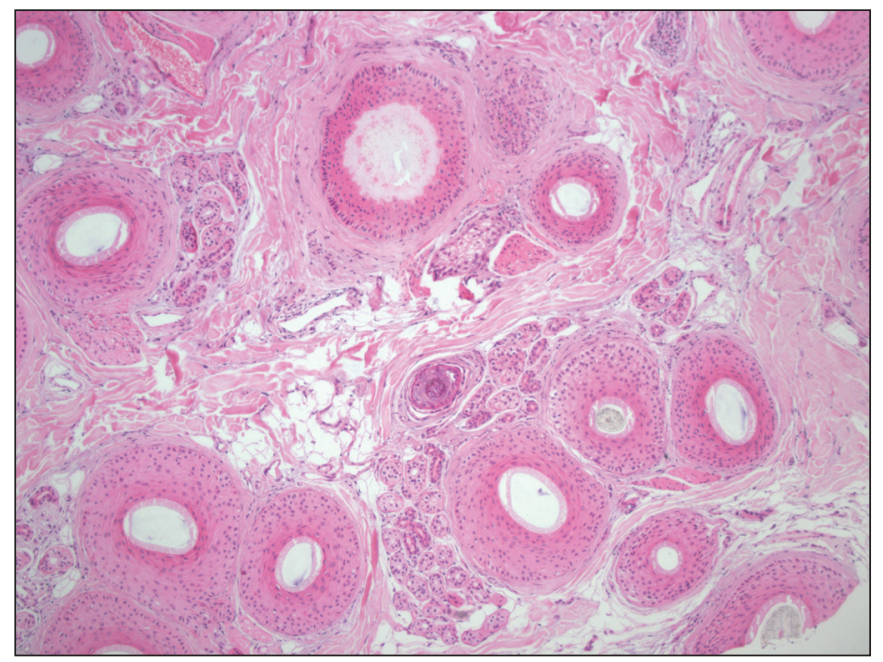

Figure 3: Androgenic alopecia: Transvers section taken at the level of dermis-subcutaneous fat tissue, displaying a mixture of hairs with various bulb depth and shaft diameter (HE;x100). spontaneous remission or exacerbations, sometimes evolving into hair loss of the entire scalp (alopecia totalis) and body hair (alopecia universalis). Histopathologically, the morphologic features change according to the duration of the episode, which is divided into three stages: acute, subacute and chronic stages (6). In early lesions, peribulber lymphocytic infiltration is the characteristic finding, which is called "swarm of bees" (Figure 4). This infiltrate may invade the follicular epithelium and the matrix, resulting in damage to matrix cells. Initially, terminal hairs are attacked, and later on vellus hairs become involved. Among

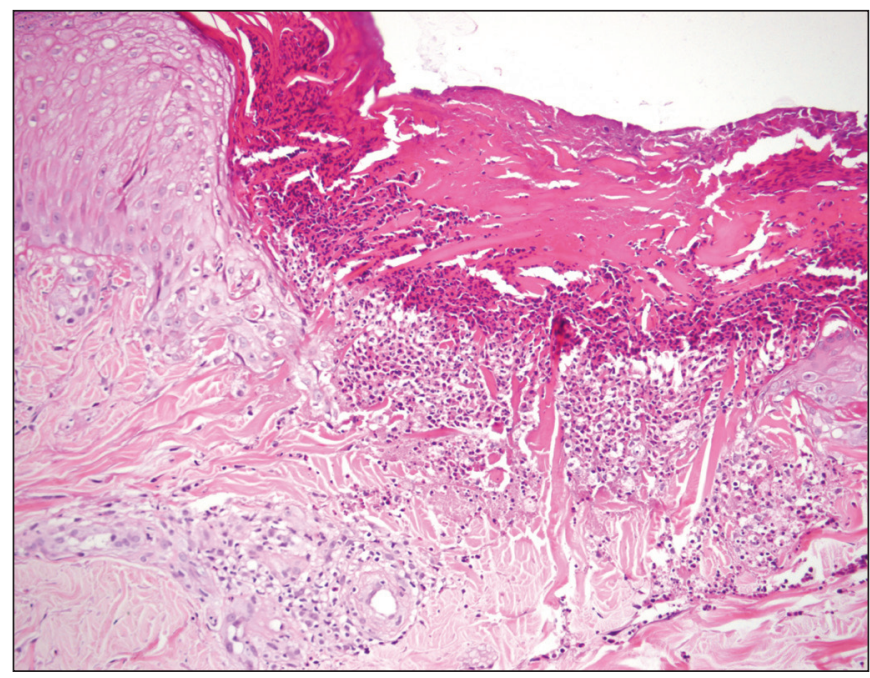

Figure 2: Acquired perforating dermatosis: In the middle of the lesion, there is an epidermal invagination filled with keratotic plug and cellular debris. Note the vertically oriented coarse collagen bundles at the base of the invagination, showing transepidermal elimination (HE; x100).

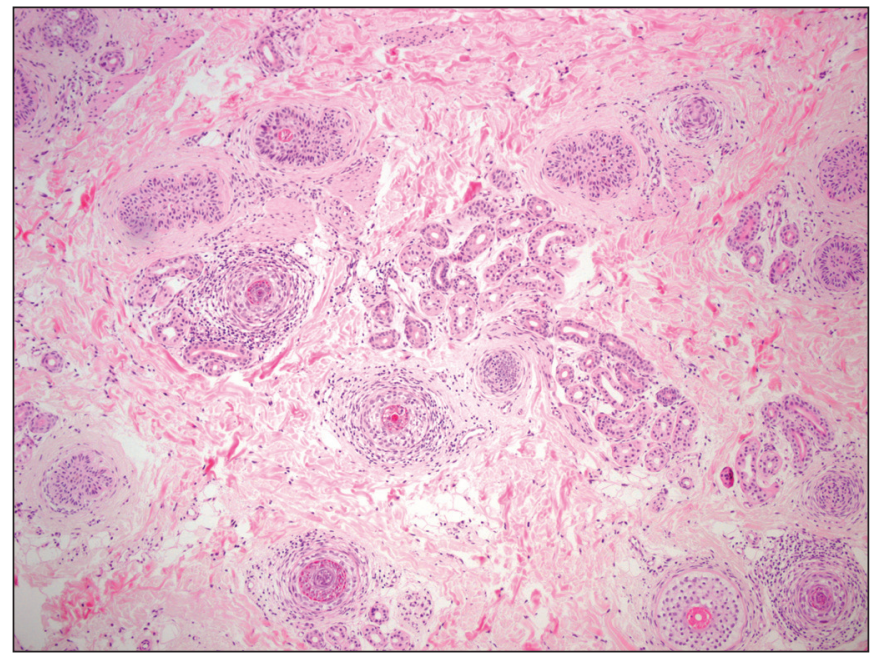

Figure 4: Alopecia areata: Peribulber lymphocytic infiltration is the characteristic finding, which is called "swarm of bees". Note that all hair follicules are miniaturized (HE;x40). 
the lymphocytes, eosinophils and also plasma cells can be seen. The anagen hair follicles shift out into catagen or telogen phases in the course of the disease, but the number of hair follicles stays unchanged for a long time. Peribulbar inflammation tends to subside after the hair has entered the catagen phase and is almost free of inflammation in the telogen phase. Subsequently, an increase in vellus hairs takes place. In the chronic stage, the majority of hair follicles will be in the catagen/telogen phase, with the presence of nanogen hair follicles. These are miniaturized, rapidly cycling hair follicles with mixed features of anagen, catagen and telogen hairs. The inner root sheath may be preserved, but many lack hair shafts, and therefore, many empty infundibula are seen. There may be perifollicular fibrosis due to the repeated attacks. Alopecia areata/universalis may also be seen as a skin finding in autoimmune polyglandular syndrome-1 (APS-1) and hypoparathyroidism.

In telogen effluvium, there is a diffuse, acute or chronic hair shedding. Histopathology of telogen efflivium reveals a normal number of hair follicles without any miniaturization, but there is an increased number of telogen hairs. In a normal scalp the ratio of telogen hairs is 5-10\%, whereas, in telogen effluvium this increases to $20-30 \%$ (5).

\section{BULLOUS DISEASES}

Multiple bullae, confined to the lower extremities may ocur in diabetic patients It is called bullosis diabeticorum (7). The bullae arise on a noninflamed base and are clear and tense. Spontaneous healing occurs within 2 to 5 weeks. The majority of these bullae are intraepidermal or subepidermal in location, with spongiotic changes in the adjacent

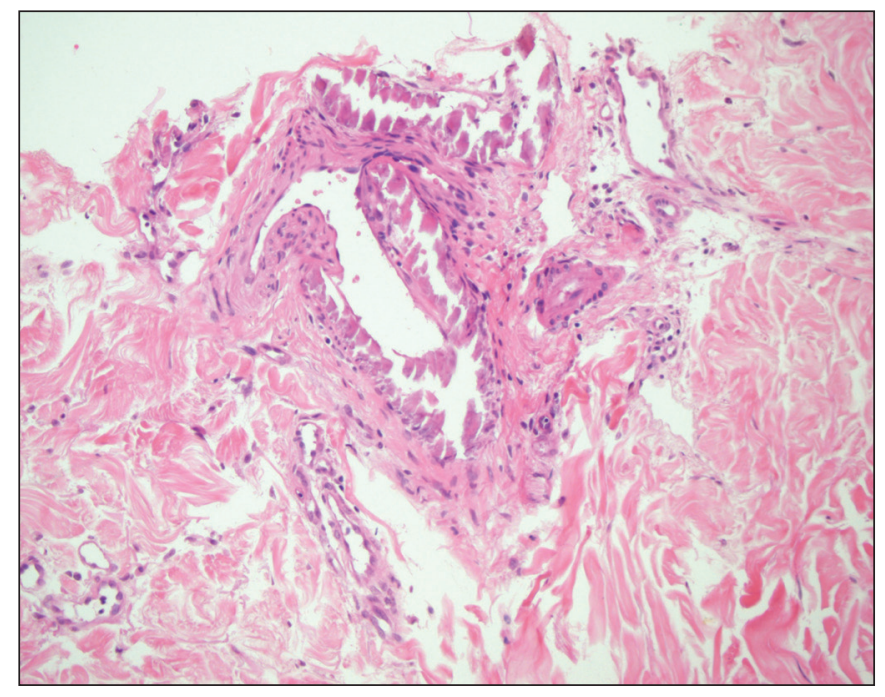

Figure 5: Calciphylaxis: Calcification of small-sized vessels in the dermis (HE; x200). epidermis. The bullae contain fibrin and a few inflammatory cells. Diabetic microangiopathy, characterized by thickenning of the walls of small blood vessels by the deposition of PAS-positive material, together with sparse perivascular lymphocytic infiltrate may accompany. Direct immunofluorescence is negative (8). Rarely, in some autoimmune thyroid diseases, especially in Graves disease, immunobullous disorders have been reported in association with it (9). These include pemphigus foliaceus, pemphigus vulgaris, bullous pemphigoid, herpes gestationis and dermatitis herpetiformis, but they are not specific for thyroid diseases. In established lesions of pemphigus, there is an acantolytic blister formation, either subcorneal or suprabasal, depending on the subtype of pemphigus. In the other above-mentioned bullous disorders, the blister is subepidermal in location.

\section{CALCIPHYLAXIS}

Calciphylaxis is usually associated with secondary or tertiary hyperparathyroidism and DM. It is a life-threatening condition, characterized by vascular calcification with thrombosis, resulting in livedo reticularis, ulceration, and gangrene, especially of fingers, toes or penis. Lesions begin as painful, necrotic, and indurated plaques and nodules that evolve into nonhealing cutaneous infarction with thick black eschars (10). Diabetic patients are more likely to develop acral gangrene (11). The characteristic microscopic feature is the calcification of the dermal and subcutaneous small-sized blood vessels, including capillaries, venules, arterioles, and small arteries, together with fibroblastic proliferation (12) (Figure 5). Endovascular thrombosis of capillaries and venules may be a feature, but not a necessary finding for the diagnosis of calciphylaxis (12). A giant cell reaction and mild inflammation can be observed in some vessel walls. The calcification of subcutaneous adipose tissue accompanied by fat necrosis is usually identified. In severe cases, calcification of subcutaneous peripheral nerves and sweat glands can be seen (13).

\section{GRANULOMA ANNULARE}

Most patients with granuloma annulare have solitary or a few oval or annular plaque lesions if they are otherwise healthy (2). Although the association of granuloma annulare with diabetes is controversial, generalized lesions are mostly considered to be among the cutaneous manifestations of DM (14). The size of the lesions may reach up to $5 \mathrm{~cm}$ and they are mostly seen on the extremities, especially on the dorsum of the hands (14). Localized lesions often resolve spontaneously, however, the generalized form has a more protracted course (2). Histopathologically, in 
classical lesions, there is focal degeneration of collagen, surrounded by histiocytes, forming palisading granulomas. In these central necrobiotic areas of collagen degeneration, increased amounts of mucin may appear as a basophilic substance between the collagen bundles (15) (Figure 6AC). Differing from necrobiosis lipoidica, the necrobiotic granulomas are present in the superficial or mid dermis. In generalized granuloma annulare, the granulomas are even situated in the papillary dermis, resembling lichen nitidus. There are variable numbers of multinucleated giant cells. A perivascular infiltrate of lymphocytes and histiocytes with some eosinophils can be detected between the granulomas, but plasma cells are rare. In the interstitial or incomplete form of granuloma annulare, there are no well-formed areas of necrobiosis, and the histiocytes are intermingled with the collagen bundles, some of which are coarse and eosinophilic in appearance, due to degeneration. This gives the dermis a "busy" look as a result of the increased number of histiocytes and lymphocytes, forming an interstitial granulomatous dermatitis.

\section{NECROBIOSIS LIPOIDICA}

Necrobiosis lipoidica appears in $0.3-1.6 \%$ of diabetic patients (2). It is stated that approximately $75 \%$ of patients with necrobiosis lipoidica have or will develop DM (4). Therefore it is also called as necrobiosis lipodica diabeticorum and is most commonly seen in middle aged adults. In patients with type 1 diabetes, necrobiosis lipoidica arise at an earlier mean age than those with type 2 diabetes (2). Women are affected more often than men. Although the exact cause of necrobiosis lipoidica is not known, it is suggested that an antibody-mediated vasculitis with secondary collagen degeneration is the main cause of this disorder (4). Other factors, such as diabetic microangiopathy, impaired neutrophil migration, hyperlipidemia, venous reflux, and abnormalities in glucose transport by fibroblasts may play role in the pathogenesis as well. The lesions of necrobiosis lipoidica are mainly located on the pretibial region and dorsum of the feet, and are typically few. It starts as an asymptomatic, red-brownish papule that evolves into a nonscaling, irregular plaque with a yellowish atrophic center, prominent superficial telengiectasia, and a slightly elevated erythematous-violaceous border (4). Histopathologically, necrobiosis lipoidica is characterized by a palisading granulomatous dermatitis with a central area of necrosis and necrobiosis (Figure 7A,B). It is mainly located in the lower parts of the dermis, accompanied by numerous plasmocytes and aggregates of lymphoid cells. Epitheloid histiocytes and a variable number of multinucleated giant cells outline the areas of necrosis. Signs of diabetic vasculopathy and even
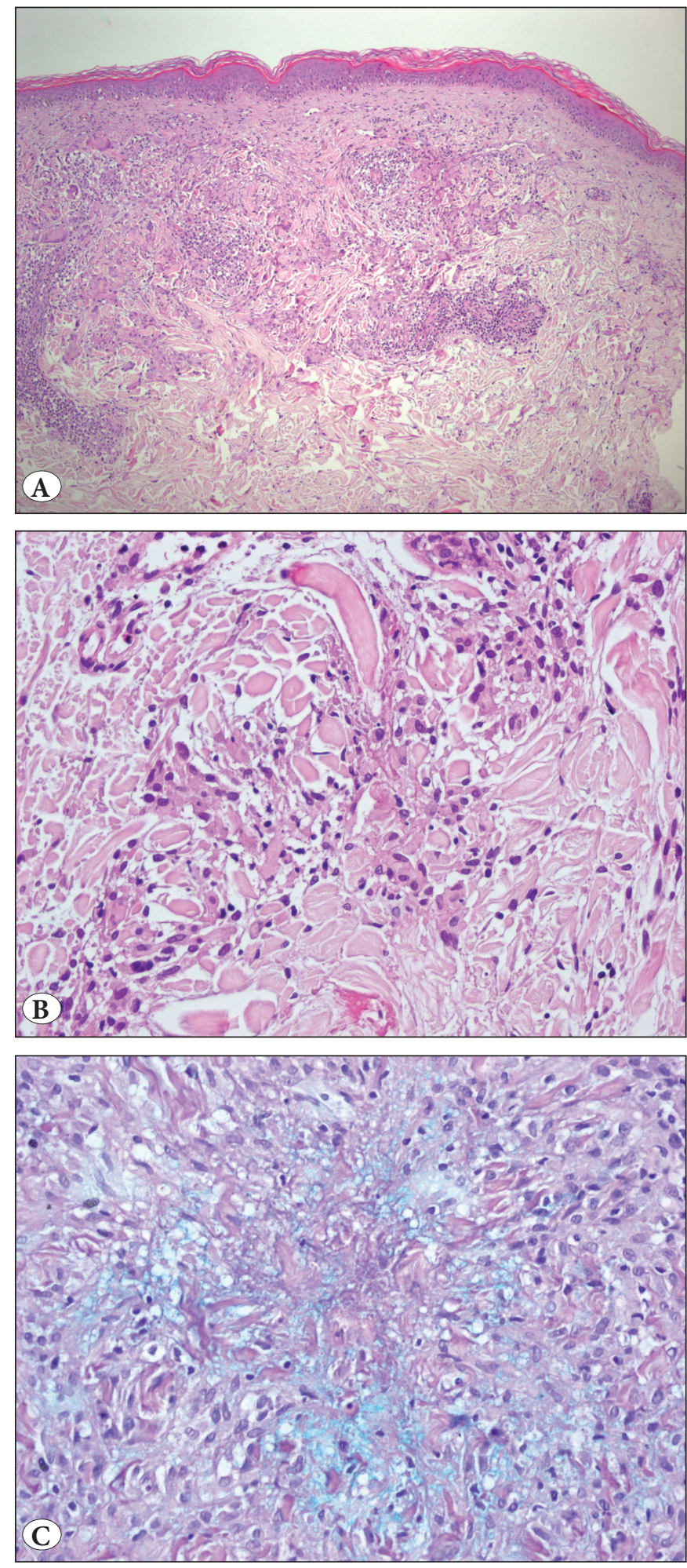

Figure 6: Granuloma annulare: A) Inflammatory cell infiltrate surrounding the areas of collagen degeneration and necrobiosis in the upper parts of the dermis (HE; x40), B) Degenerated collagen bundles, intermingled with histiocytes and some giant cells, together with an increased amounts of mucin, seen as basophilic stringy material (HE; x200), C) Colloidal iron stain can be used for the demonstration of mucin (x200). 


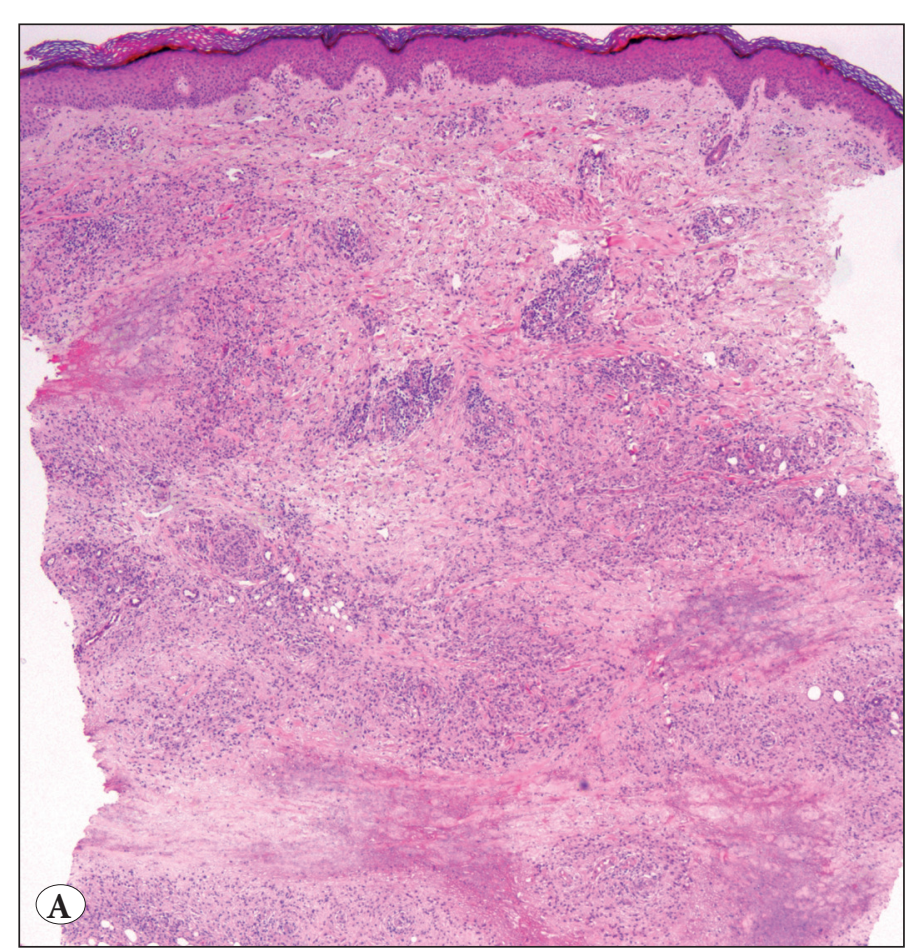

vasculitis can be seen. In the areas of necrosis, degenerated collagen fibers can be evident.

\section{NECROLYTIC MIGRATORY ERYTHEMA}

Necrolytic migratory erythema is a paraneoplastic cutaneous sign associated with a glucagon-secreting tumor, which is malignant in the majority of cases (16). The distinctive skin lesions of necrolytic migratory erythema are situated mainly on the face in perioral and perinasal distribution, the perineum, genitalia, shins, ankles, and feet. These are annular or circinate erythematous and flaccid vesiculopustular lesions, leading to crusted erosions. Various histopathological findings may be seen depending on the type of lesion that is biopsied $(17,18)$. The most distinctive histopathologic feature is the pale, vacuolated keratinocytes in the upper layers of the epidermis, ending up with necrosis, leading to subcorneal or intraepidermal cleft formation. Subcorneal pustules may be evident adjacent to necrotic areas. There is usually a broad parakeratotic scale, in which Candida may be noted.

\section{PRETIBIAL AND GENERALIZED MYXEDEMA}

Pretibial myxedema, also known as thyroid dermopathy, occasionally occurs in Hashimoto's thyroiditis. Its pathogenesis is unknown. It is frequently associated with ophthalmopathy. All patients with thyroid dermopathy have a high serum concentration of thyroid stimulating hormone (TSH) receptor antibodies, indicating an

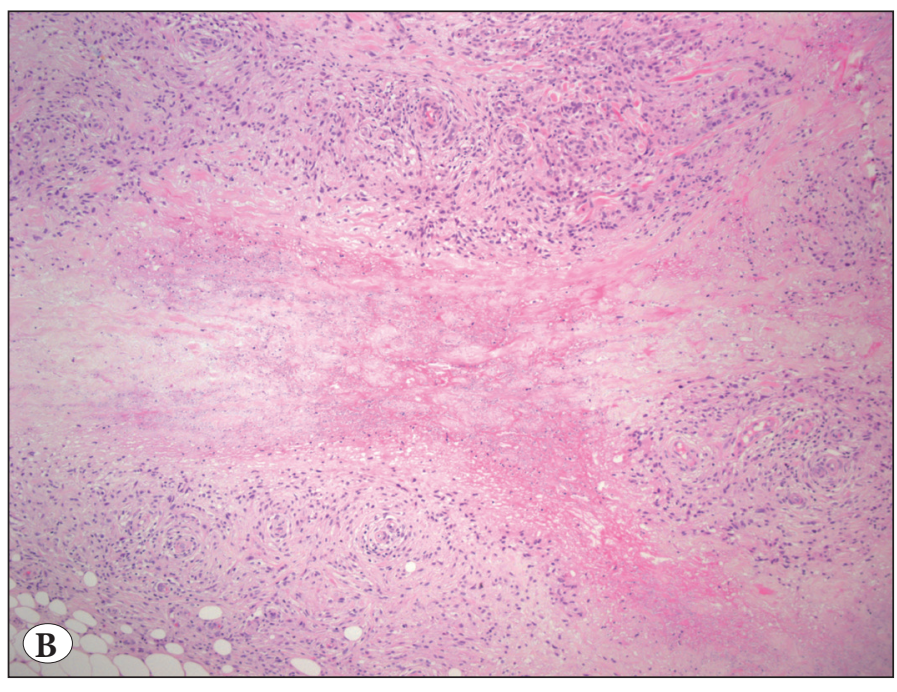

Figure 7: Necrobiosis lipoidica: A) Involvement of the whole dermis, most prominently in the lower parts (HE; $\mathrm{x} 40$ ), B) Central areas of necrosis and necrobiosis, surrounded by palisading histiocytes (HE; x200).

autoimmune condition (19). It is seen mostly on the anterior tibia and dorsa of the feet and toes, consisting of nonpitting, thickenning and induration of the skin. There may be welldemarcated pink, flesh-colored or purple-brown papules, nodules and even large masses, as well (20). Since hair follicles become prominent, a peau d'orange (orange skin) appearance may be seen. Isolated cases of involvement of the upper extremities, shoulders, upper back, and nose have been reported (19). Histopathologic examination of a biopsy specimen from a localized myxedema shows large amounts of mucin (glycosaminoglycans) deposition in the reticular dermis, particulary in the middle and deep parts, sparing the papillary dermis $(19,21)$ (Figure $8 \mathrm{~A}, \mathrm{~B})$. In hematoxylen and eosin stains, a seperation of collagen fibers can be noted. Using special stains, such as colloidal iron and alcian blue at $\mathrm{PH} 2.5$, mucin deposition can be demonstrated. There is no increase in fibroblasts, although stellate forms may be observed. Elastic fibers are reduced in number or show fragmentation. The overlying epidermis usually shows acanthosis, hyperkeratosis, follicular plugging, and sometimes papillomatosis (21). A mild superficial perivascular chronic inflammation is often present. The histopathologic features, distinguishing localized myxedema from other mucinosis or stasis dermatitis, are the lack of vascular proliferation, the absence of hemosiderin, and the preservation of the superficial dermis (19). On the other hand, the most common skin manifestation of hypothyroidism is generalized myxedema. 
The changes are most pronounced around the eyes, nose and cheeks, often resulting in a characteristic facial expression (myxedema facies) or can be seen on the distal extremities. The skin is cold and pale with widespread dryness (xerosis). Histopathologic changes of myxedematous skin are usually subtle with only a small amount of mucin within the dermis, without any fibroblastic proliferation. Sometimes, mucin deposition is present only in the perivascular and perifollicular areas.

\section{SCLEREDEMA}

Scleredema, seen in diabetic patients (scleredema diabeticorum) is characterized by remarkable thickening of the posterolateral aspect of the neck and upper back, occasionally extending to the deltoid and and lumbar region (2). There is a peau d'orange appearance of the skin with decreased sensitivity to pain and touch. It is seen in $2.5-14 \%$ of patients with poorly controled diabetes (2). However, it should be kept in mind that scleredema may occur idiopathically or due to different etiologies as well. Histopathologically, in scleredema, the dermis is markedly thickened by the replacement of subcutaneous fat by connective tissue. The collagen fibers become swollen and seperated from one another (Figure 9A,B). Various amounts of interstitial mucopolysaccharide is present. Dermal mucin can be demonstrated by the use of several stains such as alcian blue or toluidin blue ( $\mathrm{pH} 5.0$ or 7.0) or colloidal iron (4).
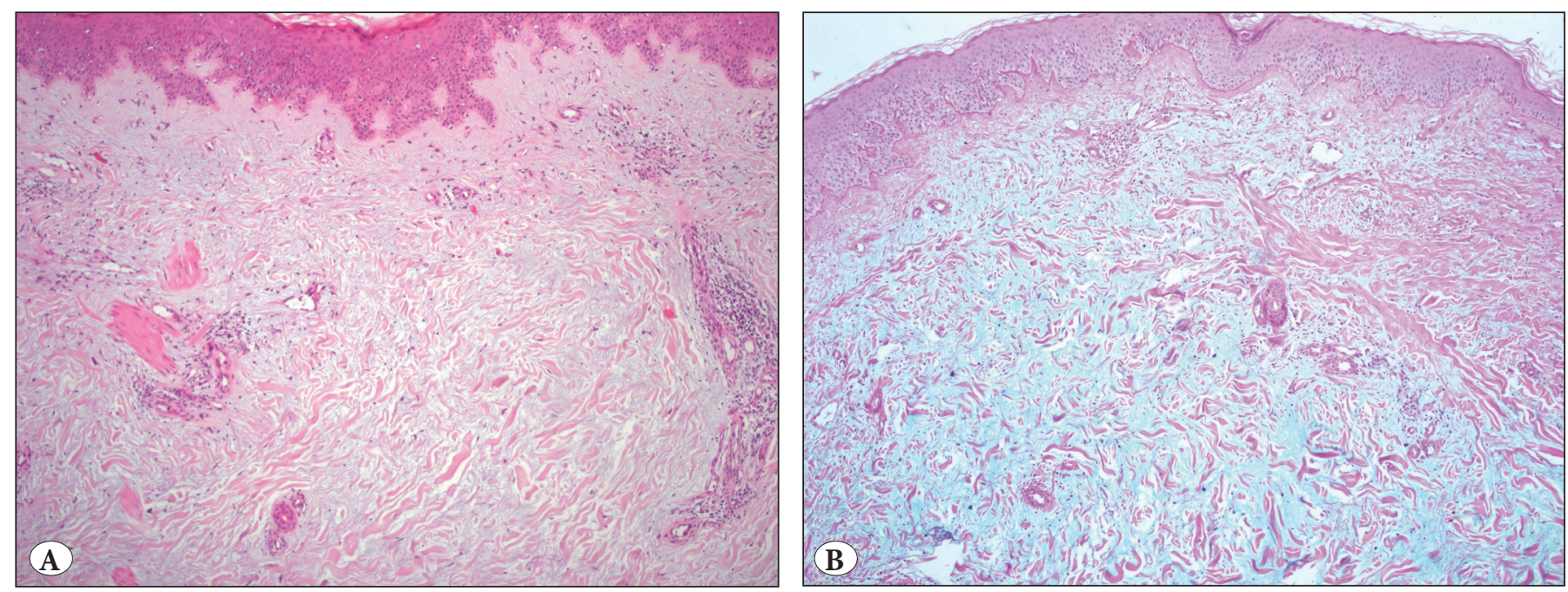

Figure 8: Pretibial myxedema: A) Collagen fibers are seperated from each other (HE; x40), B) Large amounts of mucin deposition in the reticular dermis, particulary in the middle and deep parts, sparing the papillary dermis (Colloidal iron; $\mathrm{x} 40$ ).
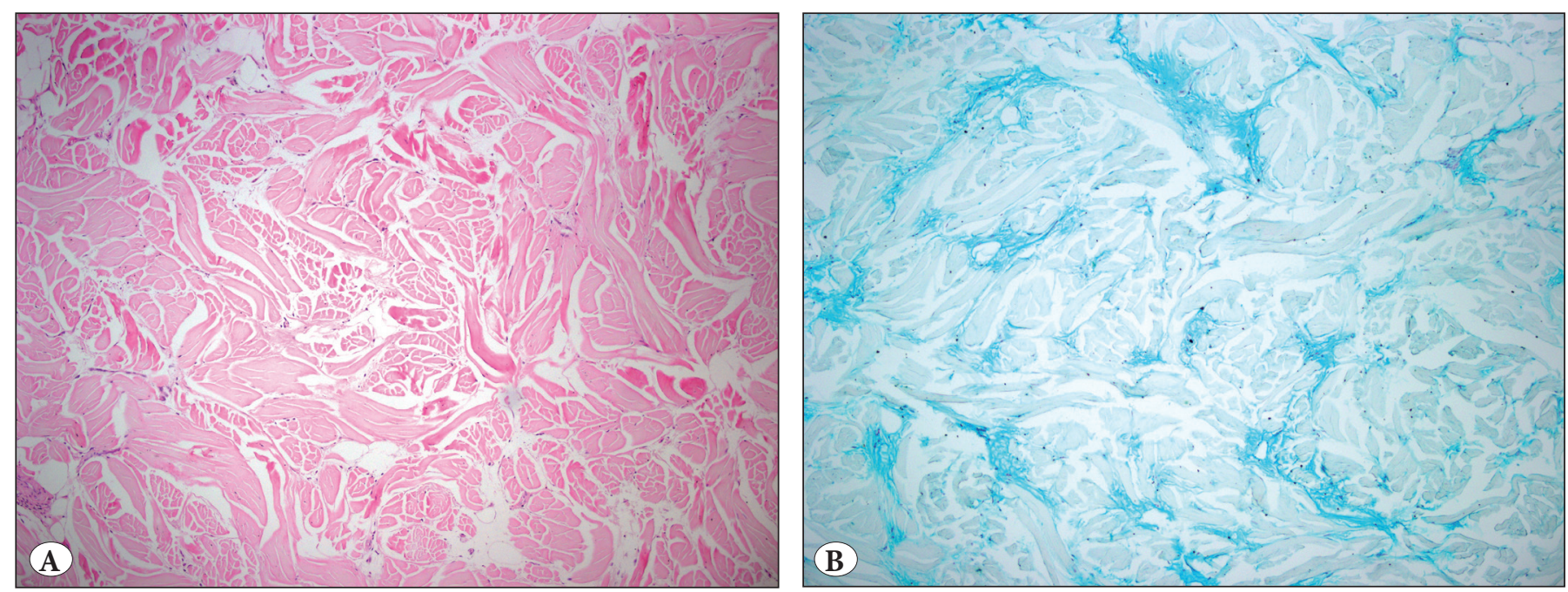

Figure 9: Scleredema: A) The dermal collagen bundles, swollen and seperated from each other (HE; x100), B)Mucin deposition, detected between the collagen bundles, using alcian blue stain (Alcian blue; x200). 


\section{SUBCUTANEOUS OSSIFICATION AND OSTEOMA CUTIS}

In pseudohypoparathyroidism, osteoma cutis can be detected as hard papules or nodules. Subcutaneous ossification may be a manifestation of Albright hereditary osteodystrophy, which is characterized by short stature, a short neck, and brachydactyly (20). Either in osteoma cutis or subcutaneous ossification in Albright hereditary osteodystrophy, small foci of ossification within the dermis or subcutaneous fat tissue are present. A circular outline is usually evident with a central space for fatty marrow. Transepidermal elimination can be detected in some cases.

\section{VITILIGO}

Vitiligo is a rare skin manifestations in APS-1, hyperthyroidism, as well as in DM type 1. From 1-7\% of all diabetic patients have vitiligo vs. $0.2-1 \%$ of the general population (2). In vitiligo, the main feature is the destruction of melanocytes in the basal layer of the epidermis. In fully developed lesions of vitiligo, the epidermis is totally devoid of melanocytes and melanin, and this is seen by using special stains, such as melanocytic markers and Fontana stain. In early lesions or at the periphery of the expanding lesions, a sparse number of melanocytes and diminished melanin pigment can still be present. Focal areas of interface or lichenoid changes may be seen especially at the periphery of the lesions.

\section{XANTOMA DISSEMINATUM}

Eruptive xanthoma is a type of xanthoma, presenting with yellow, firm, asymptomatic papules of 1 to $4 \mathrm{~mm}$ in diameter. They occur in an eruptive manner and are usually distributed on the extensor surfaces of the extremities, buttocks, and lower trunk (7). Eruptive xanthomas typically appear in association with chylomicronemia and hypertriglyceridemia, which is common in DM. Histopathologically, foamy macrophages with extravascular lipid deposits among the collagen fibers are detected in the papillary and upper reticular dermis. Neutrophils and lymphocytes are intermingled with foamy cells, but Touton type giant cells are usually absent (4). Lesions may resolve after hyperlipidemia is controlled.

\section{OTHERS}

In hypopituitarism, the cutaneous manifestations are the pallor of the skin with a yellowish tinge of the palms, soles, and nasolabial folds (carotenemia), loss of body hair, usually first involving the axillae, dry and slightly puffy skin. Relative loss of pigmentation may result due to lack of melanocytestimulating hormone (MSH). Furthermore, onycholysis, longitudinal ridging and brownish discoloration of the nail plate may occur (22).

In acromegaly, dermatologic changes are not rare but are nonspecific. Other than acanthosis nigricans, coarse facial features and a gradual enlargement of hands and feet with widened, thickenned, and stubby fingers are the typical features. Increased oiliness, widened skin pores and hypertrichosis are usually evident (23). Excess sweating is seen in $50-88 \%$ of patients with acromegaly, as a result of enhanced gland excretion rather than increased gland number (24). This may lead to abscess formation in the axillary and gluteal regions (25). Some patients may develop cutis verticis gyrata, in which the scalp becomes thickened, convoluted and furrowed, resembling the cerebral cortex.

The eruptive occurence of cherryangiomas hasbeen reported in patients with prolactinoma (26). Hyperpigmentation is detected in ectopic ACTH syndrome and less frequently in Cushing's disease, but almost never in adrenal Cushing's syndrome (25).

The secretion of the antidiuretic hormone $(\mathrm{ADH})$ in diabetes insipidus does not give rise directly to any skin changes, but its association with Langerhans cell histiocytosis and xanthoma disseminatum is well documented in literature $(27,28)$. In multiple endocrine neoplasia (MEN) 1 , facial angiofibromas, truncal collagenomas, and lipomas of various size are encountered (10). Other skin findings of MEN 1 are café-au-lait and hypopigmented macules (10). In MEN2a, primary cutaneous (macular or lichen) amyloidosis can occur (10).

The cutaneous manifestations in hypoparathyroidism, other than vitiligo and alopecia areata, are mild and nonspecific, including dry, scaly, edematous skin, rough-fragile hairs, alopecia, ungual dystrophy and nail abnormalities, such as subtle dyschromia, opaque appearance, distal onycholysis, and transvers ridges $(16,20)$. Rarely, eczematous dermatitis, hyperkeratotic and maculopapular eruptions have been reported (20). Chronic mucocutaneous candidiasis is seen mostly in APS type 1, in which autoimmune hypoparathyroidism is a component.

In DM, the skin findings can be grouped as noninfectious, infectious, vascular, neuropathic, and those related to complications of treatment $(4,7)$. Noninfectious skin manifestations can be summarized as necrobiosis lipoidica, diabetic dermopathy, granuloma annulare (especially the generalized form), diabetic thick skin, bullous diabeticorum, eruptive xanthoma, acquired perforating dermatosis, acanthosis nigricans, scleredema diabeticorum, oral lichen planus, and other rare manifestations such as "yellow 
nails and skin", vitiligo, generalized pruritus, and skin tags. Bacterial skin infections, most frequently reccurent staphylococcal pyodermas, namely fruncle and carbuncle, pseudomonas infections, such as malignant external otitis, erythrasma, and mycotic infections, such as candidiasis, particularly vulvovaginal and dermotophyte infections, are seen in $20-50 \%$ of diabetic patients as a result of poor glycemic control $(4,20)$. Rarely deep or systemic mycotic infections in diabetics include nocardiosis, cryptococcosis and zygomycoses (mainly mucormycosis). Cutaneous signs of diabetic microangiopathy are periungual telengiectasia, palmar and plantar erythema, rubeosis faciei and erysipelaslike erythema on the lower leg or dorsum of the foot. Diabetic foot ulcers, which may gradually lead to amputation of the digits or extremity, is another manifestation of vasculopathy in DM. Peripheral neuropathy, sclerosis and recurrent infections may play a role in the pathogenesis of diabetic ulcers. Skin complications due to the treatment of diabetes include maculopapular rash, generalized erythema and urticarial reactions induced by oral hypoglisemic agents, particularly first generation sulfonylureas (chlorpropamide, tolbutamide), allergic reactions due to insulin, ranging from regional erythematous reactions to angioedema, and local reactions on the injection sites, such as lipoatrophy, keloid, hyperkeratotic papules, localized pigmentation, purpuric reaction, and rarely skin abscess $(4,29)$. The association between oral lichen planus and DM has been the subject of much research (2). A significantly higher prevelance of oral lichen planus is reported in type $1 \mathrm{DM}$, compared to type 2 and a control population (30).

Skin thinning, acne, purpura, hirsutism and striae distensea are the other manifestations of Cushing's syndrome. The immunosuppressive effects of excess glucocorticoids give rise to various skin infections, such as staphylococcal, candidial and dermatophytic infections. Rare cases of deep fungal infections by aspergillus, zygomycosis and phaeohypomycosis have been reported (31).

There is an association between thyroid autoimmunity and chronic urticaria with a rate of $12-29 \%$ according to the literature (21). Asteatotic eczema, acquired ichthyosis and palmoplantar keratoderma may be seen in hypothyroidism.

In conclusion, endocrine diseases may result in changes in cutaneous function and morphology, which cause various skin manifestations, including nonspecific or pathognomonic signs. Awareness of these manifestations may permit prompt and adequate approach to the patients, and therefore facilitate the early diagnosis of the endocrine disease and even be life saving.

\section{CONFLICT OF INTEREST}

The author has declared no conflict of interest.

\section{REFERENCES}

1. Jabbour SA, Miller JL. Review article: Endocrinopathies and the skin. Int J Dermatol. 2000;39:88-99.

2. Van Hattem S, Bootsma AH, Thio HB. Skin manifestations of diabetes. Cleve Clin J Med. 2008;75:772, 774, 776-7 passim.

3. Saray Y, Seçkin D, Bilezikçi B. Acquired perforating dermatosis: Clinicopathological features in twenty-two cases. J Eur Acad Dermatol Venereol. 2006;20:679-88.

4. Rongioletti F. Pancreas disease and diabetes mellitus. In: Rongioletti F, Smoller BR, editors. Clinical and pathological aspects of skin diseases in endocrine, metabolic, nutritional and deposition disease. Berlin: Springer; 2010. 11-26.

5. Stefanato CM. Histopathology of alopecia: A clinicopathological approach to diagnosis. Histopathology. 2010; 56: 24-38.

6. Sperling LC. Alopecia areate. In: An atlas of hair pathology with clinical correlations. New York: The Parthenon Publishing Group; 2003. 63-78.

7. Oumeish OY. Skin disorders in patients with diabetes. Clin Dermatol. 2008; 26: 235-42.

8. Weedon D, Strutton G, Rubin AI. Metabolic and storage diseases. In: Weedon's skin pathology. 3rd ed. London: Churchill, Livingstone, Elsevier; 2010. 481-500.

9. Leonhardt JM, Heymann WR. Thyroid disease and the skin. Dermatol Clin. 2002; 20: 473-81.

10. Fuleihan GH, Rubeiz N. Dermatologic manifestations of parathyroid-related disorders. Clin Dermatol. 2006; 24: 281-8.

11. Hafner J, Keusch G, Wahl C, Burg G. Calciphylaxis: A syndrome of skin necrosis and acral gangrene in chronic renal failure. Vasa. 1998; 27: 137-43.

12. Wilmer WA, Magro CM. Calciphylaxis: Emerging concepts in prevention, diagnosis, and treatment. Semin Dial. 2002; 15: 172 86.

13. Molina-Ruiz AM, Cerroni L, Kutzner H, Requena L. Cutaneous deposits. Am J Dermatopathol. 2014;36:1-48.

14. Ahmed I, Goldstein B. Diabetes mellitus. Clin Dermatol. 2006 24: 237-46.

15. Weedon D, Strutton G, Rubin AI. The granulomatous reaction pattern. In: Weedon's skin pathology. 3rd ed. London: Churchill, Livingstone, Elsevier; 2010. 169-94.

16. Pujol RM1, Wang CY, el-Azhary RA, Su WP, Gibson LE, Schroeter AL. Necrolytic migratory erythema: clinicopathologic study of 13 cases. Int J Dermatol. 2004;43:12-8.

17. Kheir SM, Omura EF, Grizzle WE, Herrera GA, Lee I. Histologic variation in the skin lesions of the glucagonoma syndrome. Am J Surg Pathol. 1986; 10: 445-53

18. Kovács RK1, Korom I, Dobozy A, Farkas G, Ormos J, Kemény L. Necrolytic migratory erythema. J Cutan Pathol. 2006;33:242-5.

19. Fatourechi V. Pretibial myxedema: Pathophysiology and treatment options. Am J Clin Dermatol. 2005;6:295-309. 
20. Jabbour SA. Cutaneous manifestations of endocrine disorders: A guide for dermatologists. Am J Clin Dermatol. 2003;4:315-31.

21. Rebora A, Rongioletti F. Thyroid disease. In: Rongioletti F, Smoller $\mathrm{BR}$, editors. Clinical and pathological aspects of skin diseases in endocrine, metabolic, nutritional and deposition disease. Berlin: Springer; 2010. 27-34.

22. Davidovici BB, Orion E, Wolf R. Cutaneous manifestations of pituitary gland diseases. Clin Dermatol. 2008; 26: 288-95.

23. Centurión SA, Schwartz RA. Cutaneous signs of acromegaly. Int J Dermatol. 2002; 41: 631-4.

24. Ben-Shlomo A, Melmed S. Skin manifestations in acromegaly. Clin Dermatol. 2006; 24: 256-9.

25. Montinari M, Rongioletti F. Pituitary gland diseases. In: Rongioletti F, Smoller BR, editors. Clinical and pathological aspects of skin diseases in endocrine, metabolic, nutritional and deposition disease. Berlin: Springer; 2010. 35-42.

26. Braun-Falco O, Plewig G, Wolff HH, Burgdorf WHC. Nutritional, metabolic and endocrine disorders. In: Dermatology. 2nd ed. Berlin Heidelberg New York: Springer-Verlag; 2000. 1349-66.
27. Marchand I, Barkaoui MA, Garel C, Polak M, Donadieu J; Writing Committee. Central diabetes insipidus as the inaugural manifestation of Langerhans cell histiocytosis: Natural history and medical evaluation of 26 children and adolescents. J Clin Endocrinol Metab. 2011; 96: E1352-60.

28. Margolis S. Cutaneous xanthomatosis, diabetes insipidus, and hyperprolactinemia. Endocr Pract. 2010; 16: 933.

29. Richardson T, Kerr D. Skin-related complications of insulin therapy: Epidemiology and emerging management strategies. Am J Clin Dermatol. 2003; 4: 661-7.

30. Petrou-Amerikanou C, Markopoulos AK, Belazi M, Karamitsos D, Papanayotou P. Prevalence of oral lichen planus in diabetes mellitus according to the type of diabetes. Oral Dis. 1998; 4: 37 40.

31. Calder KB, Smoller BR. Adrenal Disease. In:Rongioletti F, Smoller $\mathrm{BR}$, editors. Clinical and pathological aspects of skin diseases in endocrine, metabolic, nutritional and deposition disease. Berlin: Springer; 2010. 3-10. 